\title{
Effectiveness of Enteral Formula with Enriched Polyunsaturated Fatty Acids in the Treatment of Anorexia Nervosa: A Pilot Open Case Study
}

\author{
Michiko Nakazato*,1,2, Shiho Arakawa ${ }^{2}$, Masayuki Takase ${ }^{2}$, Masatoshi Suzuki $^{2}$, Akihiro Shiina ${ }^{2}$, \\ Tasuku Hashimoto $^{2}$, Nobuhisa Kanahara ${ }^{2,3}$, Hiroshi Kimura ${ }^{2}$, Tomihisa Niitsu ${ }^{1}$, Taisuke Yoshida ${ }^{2}$, \\ Tetsuya Shiraishi $^{2}$, Hiroyuki Watanabe ${ }^{2}$, Tamaki Ishima ${ }^{3}$, Yuko Fujita ${ }^{3}$, Kenji Hashimoto ${ }^{3}$, Eiji \\ Shimizu $^{1,4}$ and Masaomi Iyo ${ }^{2}$
}

\author{
${ }^{1}$ Research Center for Child Mental Development, Chiba University Graduate School of Medicine, 1-8-1 Inohana, Chuo- \\ ku, Chiba City, Chiba, 260-8670, Japan \\ ${ }^{2}$ Department of Psychiatry Chiba University Graduate School of Medicine, 1-8-1 Inohana, Chuo-ku, Chiba City, Chiba, \\ 260-8670, Japan \\ ${ }^{3}$ Division of Clinical Neuroscience, Chiba University Center for Forensic Mental Health, 1-8-1 Inohana, Chuo-ku, Chi- \\ ba City, Chiba, 260-8670, Japan \\ ${ }^{4}$ Department of Cognitive Behavioral Physiology, Graduate School of Medicine, 1-8-1 Inohana, Chuo-ku, Chiba City, \\ Chiba, 260-8670, Japan
}

\begin{abstract}
Little is known about the association between polyunsaturated fatty acids (PUFAs) and eating-related pathophysiology in anorexia nervosa (AN). The aim of this study was to determine whether nutritional treatment with enteral formula, rich in PUFAs, affects (1) the concentrations of PUFA and brain-derived neurotrophic factor (BDNF) in serum, and (2) the depressive symptoms and eating-related pathophysiology in patients with AN. Thirteen patients with AN participated in this study. Serum PUFA and BDNF concentrations were measured before and after receiving the enteral formula, and eating-related psychopathology and depressive symptomatology were assessed using the eating disorder inventory-2 (EDI-2) and the Hamilton Depression Rating Scale (HDRS), respectively. Body mass index (BMI) and HDRS scores showed significant improvement after treatment. Patients receiving the nutritional treatment, enteral formula with enriched PUFAs, favorably adhered to the treatment, and showed significant improvements in BMI and depressive symptoms. Randomized controlled studies are required to further examine the effectiveness of PUFAs in AN.
\end{abstract}

Keywords: Anorexia nervosa (AN), Nutritional treatment, Polyunsaturated fatty acids (PUFAs).

\section{INTRODUCTION}

Anorexia nervosa (AN), which commonly affects adolescent or young adult women, is an eating disorder characterized by excessive attention to body weight and shape, abnormal eating behavior and low body weight. Generally, it is accompanied with various physical complications and associated with high mortality. Nutritional rehabilitation is a crucial issue in treating these patients. It is therefore important to adhere to the treatment and nutritional management, from the early stages of treatment, and remain aware of the refeeding syndrome [1].

The usefulness of a liquid formula for AN patients is well known. A previous study compared AN patients undergoing nutritional treatment with liquid formula (LF) and regular

*Address correspondence to this author at the Research Center for Child Mental Development, Chiba University Graduate School of Medicine, 1-8-1 Inohana, Chuo-ku, Chiba City, Chiba, 260-8670, Japan;Tel: +81432262975 Fax: +81432268588; E-mail: michiko.nakazato@nifty.ne.jp meals (RM) and found that the LF group did not develop a phobia of gaining weight, while the RM group exhibited a vicious cycle of gastrointestinal discomfort leading to fat phobia and stagnation in weight gain [2]. Observational studies showed that AN patients tend to be deficient in n-3 fatty acids due to limited food intake and malnutrition. Long-term deficiency in n-3 fatty acids may prolong the resolution of eating-disorder-related symptoms [3]. Recent findings suggest that diets low in long chain polyunsaturated fatty acids (LC-PUFAs) are associated with depression, anxiety, and memory dysfunction [4,5]. However, the effectiveness of nutritional therapy, involving PUFAs, for the treatment of eating-related pathophysiology as well as depressive symptoms of AN has not been fully demonstrated.

We hypothesized that enteral formula with enriched PUFAs prevents the deterioration of mood during re-feeding, and that this affects eating-related pathophysiology. The aim of the present study was to investigate the efficacy of enteral feeding enriched with PUFAs on [1] serum PUFA and serum 
BDNF concentrations, and [2] depression and eating-related pathophysiology in AN patients.

\section{PATIENTS AND METHODS}

\section{Participants}

Thirteen participants with AN including 12 female patients and 1 male patient, aged $21.7 \pm 6.7$ years (mean \pm SD) (median 20.0 years) were selected from Chiba University Hospital. They fulfilled the DSM-IV (Diagnostic and Statistical Manual; version IV, American Psychiatric Association) criteria for AN. The patients were classified as having either the restricting subtype $(n=12)$ or the binge-eating/purging subtype $(\mathrm{n}=1)$. For controls, the study utilized respective individual baseline data.

All of the subjects provided written informed consent for participation in the study. The ethics committee of Chiba University Graduate School of Medicine approved the study.

\section{Measures}

Body weight, height, and body mass index $\left(\mathrm{kg} / \mathrm{m}^{2}\right)$ of all subjects were calculated before and after nutritional treatment. All subjects completed the Eating Disorder Inventory2 (EDI-2) [6] before and after weight recovery. The EDI-2 is a 91-item, self-report questionnaire that was designed to provide a comprehensive assessment of the behavioral and psychological dimensions that are characteristic of eating disorders. Depressive symptoms were evaluated using the 17-item Hamilton Depression Rating Scale (HDRS) [7].

\section{Assessment and Treatment}

Blood samples were obtained between 11 am and 12 am and then stored at $-80^{\circ} \mathrm{C}$ until the assays were performed. Samples were taken from inpatients $(\mathrm{n}=7)$ on admission to the hospital and from outpatients $(\mathrm{n}=6)$ after initial assessment. The samples were collected again when the inpatients were discharged and when outpatients recovered after the treatment.

All inpatients received physical and psychiatric assessment according to the medical risk assessment guide for ED. Assessments included that of BMI, blood pressure, pulse rate, ECG, and musculo-skeletal assessment with squat and sit-up tests. Complete biochemical profile examination; complete blood count; differential liver function tests; and tests for determining the urea, electrolytes, serum proteins, lipid profile, creatinine kinase, glucose, transferring, and phosphate levels were also performed. The indication for inpatient admission was BMI below $13.5 \mathrm{~kg} / \mathrm{m}^{2}$ or rapid body weight loss of more than $20 \%$ body weight in 6 months. Life-threatening risks that were considered included bradycardia, hypotension, and severe proximal myopathy. Psychiatric risk factors such as severe comorbidity and suicidal tendencies were also taken into account.

All inpatients received nutritional treatment in the form of either oral or nasogastric feeding (NG) for several weeks. Table 1 shows details of the composition of the enteral formula, which was rich in omega-3 fatty acids. Dietary supplement formula, rich in omega-3 PUFA, was provided 3 times a day with the initial dose being $600 \mathrm{kcal} / \mathrm{day}$; the total
Table 1. Full Details of the Composition of the Enteral Formula with PUFAs

\begin{tabular}{|c|c|c|c|c|}
\hline \multirow{2}{*}{$\begin{array}{l}\text { Composition } \\
\text { Protein }\end{array}$} & \multicolumn{2}{|c|}{$\begin{array}{l}200 \mathrm{~mL} \text { Pouch } \\
\text { (200 kcal) }\end{array}$} & \multicolumn{2}{|c|}{$\begin{array}{c}1000 \mathrm{~mL} \\
(1000 \text { kcal) }\end{array}$} \\
\hline & 8.76 & $\mathrm{~g}$ & 43.8 & $\mathrm{~g}$ \\
\hline Fat & 4.46 & $\mathrm{~g}$ & 22.3 & $\mathrm{~g}$ \\
\hline Carbohydrate & 31.24 & $\mathrm{~g}$ & 156.2 & $\mathrm{~g}$ \\
\hline \multirow[t]{2}{*}{$\mathrm{Na}$} & 147.6 & $\mathrm{mg}$ & 738.0 & $\mathrm{mg}$ \\
\hline & $(6.4$ & $\mathrm{mEq})$ & $(32.0$ & $\mathrm{mEq})$ \\
\hline \multirow[t]{2}{*}{$\mathrm{K}$} & 276 & $\mathrm{mg}$ & 1380 & $\mathrm{mg}$ \\
\hline & $(7.0$ & $\mathrm{mEq})$ & $(35.0$ & $\mathrm{mEq})$ \\
\hline \multirow[t]{2}{*}{$\mathrm{Ca}$} & 88.0 & $\mathrm{mg}$ & 440.0 & $\mathrm{mg}$ \\
\hline & $(4.4$ & $\mathrm{mEq})$ & $(22.0$ & $\mathrm{mEq})$ \\
\hline \multirow[t]{2}{*}{$\mathrm{Mg}$} & 38.6 & $\mathrm{mg}$ & 193.0 & $\mathrm{mg}$ \\
\hline & $(3.2$ & $\mathrm{mEq})$ & $(16.0$ & $\mathrm{mEq})$ \\
\hline $\mathrm{P}$ & 88.0 & $\mathrm{mg}$ & 440.0 & $\mathrm{mg}$ \\
\hline \multirow[t]{2}{*}{$\mathrm{Cl}$} & 234 & $\mathrm{mg}$ & 1170 & $\mathrm{mg}$ \\
\hline & $(6.6$ & $\mathrm{mEq})$ & $(33.0$ & $\mathrm{mEq})$ \\
\hline $\mathrm{Fe}$ & 1,250 & $\mu \mathrm{g}$ & 6,250 & $\mu \mathrm{g}$ \\
\hline $\mathrm{Zn}$ & 1,280 & $\mu \mathrm{g}$ & 6,400 & $\mu \mathrm{g}$ \\
\hline $\mathrm{Mn}$ & 266 & $\mu \mathrm{g}$ & 1330 & $\mu \mathrm{g}$ \\
\hline $\mathrm{Cu}$ & 250 & $\mu \mathrm{g}$ & 1250 & $\mu \mathrm{g}$ \\
\hline Retinol palmitate & 414 & IU & 2070 & IU \\
\hline Cholecalciferol & 27.2 & IU & 136.0 & IU \\
\hline Tocopherol acetate & 1,300 & $\mu \mathrm{g}$ & 6,500 & $\mu g$ \\
\hline Phytonadione & 125.0 & $\mu \mathrm{g}$ & 625.0 & $\mu \mathrm{g}$ \\
\hline Thiamin & 760 & $\mu g$ & 3,800 & $\mu \mathrm{g}$ \\
\hline Riboflavin & 490 & $\mu \mathrm{g}$ & 2450 & $\mu \mathrm{g}$ \\
\hline Pyridoxine & 750 & $\mu \mathrm{g}$ & 3,750 & $\mu \mathrm{g}$ \\
\hline Cyanocobalamin & 0.640 & $\mu \mathrm{g}$ & 3.200 & $\mu \mathrm{g}$ \\
\hline Ascorbic acid & 56.2 & $\mathrm{mg}$ & 281.0 & $\mathrm{mg}$ \\
\hline Nicotinamide & 5.00 & $\mathrm{mg}$ & 25.00 & $\mathrm{mg}$ \\
\hline Pantothenic acid & 1,916 & $\mu \mathrm{g}$ & 9,580 & $\mu \mathrm{g}$ \\
\hline Folic acid & 75.0 & $\mu g$ & 375.0 & $\mu \mathrm{g}$ \\
\hline Biotin & 7.72 & $\mu \mathrm{g}$ & 38.6 & $\mu \mathrm{g}$ \\
\hline \multicolumn{5}{|l|}{ Specifically } \\
\hline Tricaprilin & 1,500 & $\mathrm{mg}$ & 7,500 & $\mathrm{mg}$ \\
\hline Linoleic acid & 900 & $\mathrm{mg}$ & 4,500 & $\mathrm{mg}$ \\
\hline$\alpha$-Linolenic acid & 300 & $\mathrm{mg}$ & 1,500 & $\mathrm{mg}$ \\
\hline
\end{tabular}

PUFAs; polyunsaturated fatty acids

volume was gradually increased to a maximum volume of $2400 \mathrm{kcal} /$ day. Weekly body weight gain was set at $0.5-1.0$ $\mathrm{kg}$, and body weight was estimated once a week before breakfast. Psychological care, family counseling, and nutritional counseling were provided for the participants. 


\section{Serum Concentrations of BDNF and PUFA}

The serum samples from the participants were collected between $11 \mathrm{am}$ and $12 \mathrm{pm}$, and were stored at $-80^{\circ} \mathrm{C}$ until the assays were performed. Serum BDNF was measured using an Emax immunoassay system kit (Promega; Madison, WI, USA). In brief, 96-well plates were coated with anti-BDNF monoclonal antibodies and incubated at $4^{\circ} \mathrm{C}$ for $18 \mathrm{~h}$, after which the plates were incubated in a blocking buffer $(1 \mathrm{~h}$, room temperature). The samples and BDNF standards were maintained at room temperature with shaking for $2 \mathrm{~h}$, and were then washed with buffers. Plates were incubated with anti-human BDNF polyclonal antibodies $(2 \mathrm{~h}$, room temperature), washed, and incubated with anti-immunoglobulin (Ig) $\mathrm{Y}$ antibodies that were conjugated with horseradish peroxidase $(1 \mathrm{~h}$, room temperature). The plates were then treated with peroxidase substrate and tetramethylbenzidine solution to produce a color reaction. This reaction was stopped with 1 $M$ hydrochloric acid and the absorbance at $450 \mathrm{~nm}$ was measured with an automated microplate reader (Emax; Molecular Devices, Sunnyvale, CA, USA). Serum PUFA concentrations were measured using gas-chromatography.

\section{Statistical Analysis}

The data are presented as the mean standard deviation (SD). Statistical analyses were performed using the paired $t$ test (before and after weight recovery). Calculations were performed using the statistical software package SPSS 20.0 for windows (SPSS 20.0; Chicago, IN, USA). Differences with $\mathrm{p}<0.05$ were considered statistically significant.

\section{RESULTS}

\section{Adherence to the Study and Beneficial Effects}

All 13 patients with acute AN who were treated with enteral formula favorably adhered to the treatment without any complications. The average daily dose of enteral formula during treatment was $28.8 \pm 11.1 \mathrm{kcal} / \mathrm{kg} / \mathrm{day}$. The formula contains $1500 \mathrm{mg} / 1000 \mathrm{ml} / \mathrm{kcal}$ of $\alpha$-linolenic acid and 4500 $\mathrm{mg} / 1000 \mathrm{ml} / \mathrm{kcal}$ of linoleic acid (Table 1). The average duration of nutritional treatment formula was $47.1 \pm 33.7$ days (mean $\pm \mathrm{SD}$ ). No antipsychotic drugs were administered to patients during the treatment. There were no side effects in any patient.

\section{Changes in Physical and Psychological Measures and Serum PUFAs between Baseline and After Nutritional Treatment}

Table 2 shows the changes in physical and psychological measures and the concentrations of serum PUFAs before and after enteral formula treatment. In all subjects, BMI was 14.1 $\pm 1.5 \mathrm{~kg} / \mathrm{m}^{2}$ (mean $\pm \mathrm{SD}$ ) before and after partial recovery $\left[15.3 \pm 1.8 \mathrm{~kg} / \mathrm{m}^{2}(\right.$ mean $\left.\pm \mathrm{SD})\right]$. There were significant differences between the BMI of patients before and after nutritional treatment $(p=0.002)$. Moreover, there was a significant improvement in HDRS scores before and after treatment $(\mathrm{p}=0.003)$. In patients with the restricting subtype $(\mathrm{n}=12)$, BMI was $14.2 \pm 1.5 \mathrm{~kg} / \mathrm{m}^{2}$ (mean $\pm \mathrm{SD}$ ) before and after partial recovery $\left[15.4 \pm 1.9 \mathrm{~kg} / \mathrm{m}^{2}(\right.$ mean $\left.\pm \mathrm{SD})\right]$, indicating a significant improvement in BMI after nutritional treatment $(\mathrm{p}=0.013)$. There was a significant improvement in the HDRS scores after treatment $(p=0.007)$. There were no significant differences in the serum PUFA or BDNF concentrations between baseline and after partial weight gain among the subjects and the restricted subtype subjects.

\section{DISCUSSIONS}

In the present study, all 13 patients with acute-phase AN, who were treated with enteral formula containing PUFAs, exhibited favorable adherence to treatment without any complications. Although treatment duration was relatively short

Table 2. Changes in Physical and Psychological Measures, Serum PUFAs before and after Enternal Formula Treatment

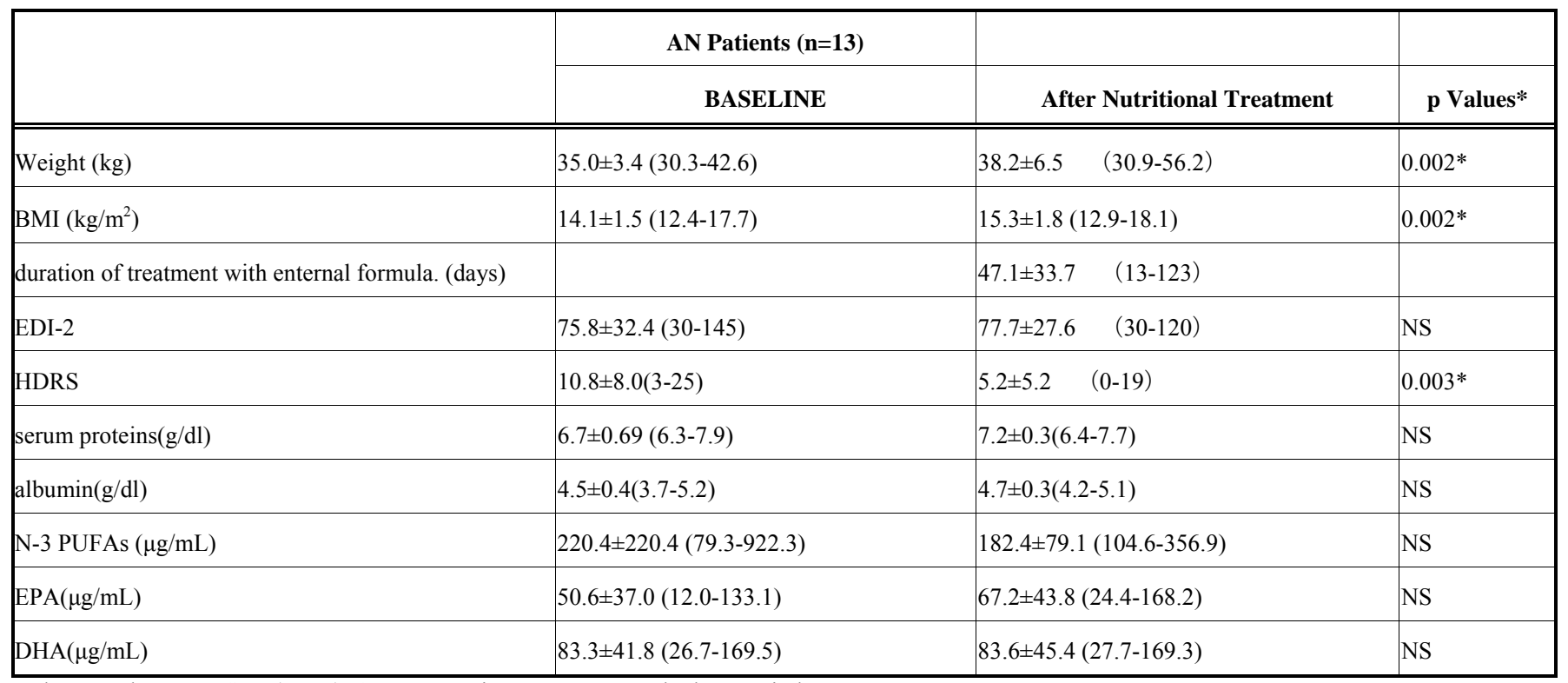

Values are the mean \pm S.D. (range). AN $=$ anorexia nervosa, $\mathrm{BMI}=$ body mass index,

EDI-2 = Eating Disorder Inventory-2, HDRS = Hamilton Depression Rating Scale, PUFAs=poly-unsaturated fatty acids

$\mathrm{EPA}=$ eicosapentaenoic acid, DHA=docosahexaenoic acid. 
(47 days), improvements in BMI and depressive symptoms (as evaluated by the HDRS) were observed. There were no significant changes in the serum concentrations of PUFAs between baseline and after nutritional treatment.

Several studies have examined the use of nutritional formula enriched with PUFAs for the treatment of AN. One study reported favorable prognosis in 3 of 7 patients treated with an EPA supplement of 1g/day for 3 months [8]. Nine AN patients were found to be deficient in essential fatty acids due to limited food intake, and they developed PUFA disorders frequently that lead to physical complications, mood swings, and distorted cognitive functions. Comorbid symptoms are thought to be related to disorders of unsaturated fatty acids. These symptoms include gastrointestinal and dermatologic symptoms, osteoporosis, circulatory failure, infection, and depressive symptoms [2].

In this study, serum PUFA concentrations did not increase after the supplement was given. We assume that there were 2 reasons for this insignificant effect on EPA. First, as shown in Table 2, there was a large variation between individuals and a small sample size. Second, a previous study suggested that AN patients displayed a complex membrane FA profile with deficits in membrane LA-PUFA, which might affect the tolerance to elevation of serum PUFA concentrations in such participants [9]. Further, serum BDNF did not change following treatment. We assume that one of the reasons for this insignificant change in BDNF was because nutritional treatment was provided only for a short period of time and BMIs of the patients were still increasing. In our previous study, serum BDNF concentrations did not change after partial weight recovery in AN patients [10]. In animal studies, DHA-treated rats exhibited higher levels of spatial learning ability and BDNF concentrations than the untreated rats [11]. It has therefore been suggested that exercises and administration of PUFAs can influence synaptic plasticity via BDNF and thereby improve cognitive function $[11,12]$. The nutritional treatment with PUFAs may contribute to deteriorating mood, which is a common feature of re-feeding, and increases the risk of relapse, as well as improvement of cognitive impairments [13]. Further research is needed to clarify the relationship between the concentrations of serum PUFA and serum BDNF.

The limitations of the present study include the following: small sample size, the lack of comparison with a healthy control group, the short duration of nutritional treatment, and different treatment durations among different patients. Moreover, it was not a randomized controlled trial.

\section{CONCLUSION}

Nutritional treatment with enteral formula containing PUFAs achieved favorable adherence to treatment and improvements in BMI and depressive symptoms without any drop-out. No significant change was observed in serum PUFA concentrations after nutritional treatment. Further studies are needed to clarify the involvement of PUFAs in the pathology of AN.

\section{ACKNOWLEDGEMENTS}

We would thank all patients for participating in this study and multidisciplinary team for providing treatment.

\section{DECLARATION OF INTEREST}

The authors confirm that this article content has no conflicts of interest.

\section{REFERENCES}

[1] Treasure J, Schmidt U, Van Furth E, Eds. Handbook of Eating Disorders, $2^{\text {nd }}$ ed. Sussex: Wiley 2003.

[2] Arii I, Yamashita T, Kinoshita M, et al. Treatment for inpatients with anorexia nervosa: comparison of liquid formula with regular meals for improvement from emaciation. Psychiatry Clin Neurosci 1996; 50: 55-9.

[3] Ayton AK. Dietary polyunsaturated fatty acids and anorexia nervosa: is there a link? Nutr Neurosci 2004; $7: 1$-12.

[4] Chiu CC, Frangou S, Chang CJ, et al. Associations between n-3 PUFA concentrations and cognitive function after recovery from late-life depression. Am J Clin Nutr 2012; 95: 420-7.

[5] Freeman MP, Hibbeln JR, Wisner KL, et al. Omega-3 fatty acids: evidence basis for treatment and future research in psychiatry. $J$ Clin Psychiatry 2006; 67: 1954-67.

[6] Garner DM. Manual for the Eating Disorder Inventory (EDI), Odessa, FL: Psychological Assessment Resources 1991.

[7] Hamilton M. A rating scales for depression. J Neurol Neurosurg Psychiatr 1960; 23: 56-62.

[8] Ayton AK, Azaz A, Horrobin DF. A pilot open case series of ethylEPA supplementation in the treatment of anorexia nervosa. Prostaglandins Leukot Essent Fatty Acids 2004; 71: 205-9.

[9] Caspar-Bauguil S, Montastier E, Galinon F, et al. Anorexia nervosa patients display a deficit in membrane long chain poly-unsaturated fatty acids. Clin Nutr 2012; 3131: 386-90.

[10] Nakazato M, Hashimoto K, Yoshimura K, et al. No change between the serum brain-derived neurotrophic factor in female patients with anorexia nervosa before and after partial weight recovery. Prog Neuropsychopharmacol Biol Psychiatry 2006; 30: 1117 21.

[11] Wu A, Ying Z, Gomez-Pinilla F. Docosahexaenoic acid dietary supplementation enhances the effects of exercise on synaptic plasticity and cognition. Neuroscience 2008; 155: 751-9.

[12] Venna VR, Deplanque D, Allet C, et al. PUFA induce antidepressant-like effects in parallel to structural and molecular changes in the hippocampus. Psychoneuroendocrinology 2009; 34: 199-211.

[13] Boucher O, Burden MJ, Muckle G, et al. Neurophysiologic and neurobehavioral evidence of beneficial effects of prenatal omega-3 fatty acid intake on memory function at school age. Am J Clin Nutr 2011; 93: 1025-37. 